PROCEEDINGS OF THE

AMERICAN MATHEMATICAL SOCIETY

Volume 128, Number 1, Pages 119-123

S 0002-9939(99)04965-5

Article electronically published on July 6, 1999

\title{
INVERTIBLE COMPLETIONS OF $2 \times 2$ UPPER TRIANGULAR OPERATOR MATRICES
}

\author{
JIN KYU HAN, HONG YOUL LEE, AND WOO YOUNG LEE \\ (Communicated by Palle E. T. Jorgensen)
}

Abstract. In this note we prove that if

$$
M_{C}=\left(\begin{array}{cc}
A & C \\
0 & B
\end{array}\right)
$$

is a $2 \times 2$ upper triangular operator matrix acting on the Banach space $X \oplus Y$, then $M_{C}$ is invertible for some $C \in \mathcal{L}(Y, X)$ if and only if $A \in \mathcal{L}(X)$ and $B \in \mathcal{L}(Y)$ satisfy the following conditions:

(i) $A$ is left invertible;

(ii) $B$ is right invertible;

(iii) $X / A(X) \cong B^{-1}(0)$.

Furthermore we show that $\sigma(A) \cup \sigma(B)=\sigma\left(M_{C}\right) \cup W$, where $W$ is the union of certain of the holes in $\sigma\left(M_{C}\right)$ which happen to be subsets of $\sigma(A) \cap \sigma(B)$.

\section{INTRODUCTION}

Du and Pan [1] have recently considered the invertible completions of partially specified $2 \times 2$ upper triangular operator matrices. Their main result can be described as follows: if $M_{C}=\left(\begin{array}{cc}A & C \\ 0 & B\end{array}\right)$ is an operator acting on the Hilbert space $H \oplus K$, then

$$
\bigcap_{C \in \mathcal{L}(K, H)} \sigma\left(M_{C}\right)=\sigma_{l}(A) \cup \sigma_{r}(B) \cup\{\lambda \in \mathbb{C}: n(B-\lambda) \neq d(A-\lambda)\},
$$

where $\sigma(\cdot), \sigma_{l}(\cdot), \sigma_{r}(\cdot), n(\cdot)$, and $d(\cdot)$ denote the spectrum, the left spectrum, the right spectrum, the nullity, and the deficiency, respectively. In this note we extend the above result to Banach spaces and show that the passage from $\sigma(A) \cup \sigma(B)$ to $\sigma\left(M_{C}\right)$ is the punching of some open sets in $\sigma(A) \cap \sigma(B)$.

Let $\mathcal{L}(X, Y)$ denote the set of bounded linear operators from a Banach space $X$ to a Banach space $Y$ and abbreviate $\mathcal{L}(X, X)$ to $\mathcal{L}(X)$. Recall [3] that $T \in \mathcal{L}(X, Y)$ is called regular if there is an operator $T^{\prime} \in \mathcal{L}(Y, X)$ for which

$$
T=T T^{\prime} T
$$

then $T^{\prime}$ is called a generalized inverse for $T$. If $T \in \mathcal{L}(X, Y)$ is left or right invertible, then, evidently, $T$ is regular: in this case a left or right inverse is just a generalized

Received by the editors October 26, 1996 and, in revised form, March 10, 1998.

1991 Mathematics Subject Classification. Primary 47A10, 47A55.

Key words and phrases. Spectrum, regular, $2 \times 2$ upper triangular operator matrices.

This work was partially supported by BSRI 96-1420 and KOSEF 94-0701-02-01-3. 
inverse. Also if $T \in \mathcal{L}(X, Y)$ is regular with a generalized inverse $T^{\prime}$, then $X$ and $Y$ can be decomposed as follows (cf. [3]):

$$
T^{-1}(0) \oplus T^{\prime} T(X)=X \quad \text { and } \quad T(X) \oplus\left(T T^{\prime}\right)^{-1}(0)=Y .
$$

When $A \in \mathcal{L}(X)$ and $B \in \mathcal{L}(Y)$ are given we denote by $M_{C}$ an operator acting on $X \oplus Y$ of the form

$$
M_{C}=\left(\begin{array}{cc}
A & C \\
0 & B
\end{array}\right)
$$

where $C \in \mathcal{L}(Y, X)$.

\section{A NECESSARY AND SUfFICIENT CONDition FOR INVERTIBILITy OF $M_{C}$}

We begin with:

Lemma 1. If $A \in \mathcal{L}(X)$ and $B \in \mathcal{L}(Y)$ are both invertible, then $M_{C}$ is invertible for every $C \in \mathcal{L}(Y, X)$.

Proof. The inverse of $M_{C}$ is $\left(\begin{array}{cc}A^{-1}-A^{-1} C B^{-1} \\ 0 & B^{-1}\end{array}\right)$.

The following is our main result. To prove the necessary condition of the invertibility of $M_{C}$ we need the "ghost" of an index theorem due to Harte [4]:

Theorem 2. A $2 \times 2$ operator matrix $M_{C}$ is invertible for some $C \in \mathcal{L}(Y, X)$ if and only if $A \in \mathcal{L}(X)$ and $B \in \mathcal{L}(Y)$ satisfy the following conditions:

(i) $A$ is left invertible;

(ii) $B$ is right invertible;

(iii) $X / A(X) \cong B^{-1}(0)$.

Proof. Suppose $M_{C}=\left(\begin{array}{cc}A & C \\ 0 & B\end{array}\right)$ is invertible for some $C \in \mathcal{L}(Y, X)$ and write

$$
M_{C}=\left(\begin{array}{cc}
I & 0 \\
0 & B
\end{array}\right)\left(\begin{array}{cc}
I & C \\
0 & I
\end{array}\right)\left(\begin{array}{cc}
A & 0 \\
0 & I
\end{array}\right) .
$$

Then $A$ is left invertible and $B$ is right invertible. On the other hand since $\left(\begin{array}{ll}I & 0 \\ 0 & B\end{array}\right)\left(\begin{array}{ll}I & C \\ 0 & I\end{array}\right)$ and $\left(\begin{array}{cc}A & 0 \\ 0 & I\end{array}\right)$ are both regular, an index theorem of Harte ([4, Theorem 2]) gives

$$
\begin{aligned}
& \operatorname{ker}\left(\begin{array}{cc}
A & 0 \\
0 & I
\end{array}\right) \times \operatorname{ker}\left(\left(\begin{array}{ll}
I & 0 \\
0 & B
\end{array}\right)\left(\begin{array}{cc}
I & C \\
0 & I
\end{array}\right)\right) \times\left(\begin{array}{l}
X \\
Y
\end{array}\right) / \operatorname{ran} M_{C} \\
& \cong \operatorname{ker} M_{C} \times\left(\begin{array}{l}
X \\
Y
\end{array}\right) / \operatorname{ran}\left(\begin{array}{cc}
A & 0 \\
0 & I
\end{array}\right) \times\left(\begin{array}{l}
X \\
Y
\end{array}\right) / \operatorname{ran}\left(\left(\begin{array}{ll}
I & 0 \\
0 & B
\end{array}\right)\left(\begin{array}{ll}
I & C \\
0 & I
\end{array}\right)\right)
\end{aligned}
$$

which implies

$$
A^{-1}(0) \times B^{-1}(0) \times\{0\} \cong\{0\} \times X / A(X) \times Y / B(Y),
$$

which gives that $B^{-1}(0) \cong X / A(X)$ because $A$ is left invertible and $B$ is right invertible.

For the converse observe that if $A^{\prime}$ is a left inverse of $A$ and if $B^{\prime}$ is a right inverse of $B$, then, as in (0.2), $X$ and $Y$ can be decomposed as

$$
A(X) \oplus\left(A A^{\prime}\right)^{-1}(0)=X \quad \text { and } \quad B^{-1}(0) \oplus B^{\prime} B(Y)=Y .
$$


Then, by (iii), we have that $\left(A A^{\prime}\right)^{-1}(0) \cong B^{-1}(0)$. Thus there is an isomorphism $J: B^{-1}(0) \rightarrow\left(A A^{\prime}\right)^{-1}(0)$. Define an operator $C: Y \rightarrow X$ by

$$
C:=\left(\begin{array}{cc}
J & 0 \\
0 & 0
\end{array}\right):\left(\begin{array}{c}
B^{-1}(0) \\
B^{\prime} B(Y)
\end{array}\right) \longrightarrow\left(\begin{array}{c}
\left(A A^{\prime}\right)^{-1}(0) \\
A(X)
\end{array}\right) .
$$

Then we have that $C \in \mathcal{L}(Y, X), C(Y)=\left(A A^{\prime}\right)^{-1}(0)$ and $C^{-1}(0)=B^{\prime} B(Y)$. We now claim that $M_{C}$ is one-one and onto, and hence invertible. Indeed we have

$$
\left(\begin{array}{ll}
A & C \\
0 & B
\end{array}\right)\left(\begin{array}{l}
X \\
Y
\end{array}\right)=\left(\begin{array}{c}
A(X)+C(Y) \\
B(Y)
\end{array}\right)=\left(\begin{array}{c}
A(X)+\left(A A^{\prime}\right)^{-1}(0) \\
Y
\end{array}\right)=\left(\begin{array}{l}
X \\
Y
\end{array}\right)
$$

and

$$
\left(\begin{array}{ll}
A & C \\
0 & B
\end{array}\right)\left(\begin{array}{l}
x \\
y
\end{array}\right)=\left(\begin{array}{l}
0 \\
0
\end{array}\right) \Longrightarrow\left\{\begin{array} { l } 
{ A x + C y = 0 } \\
{ B y = 0 }
\end{array} \Longrightarrow \left\{\begin{array}{l}
A x=0 \\
B y=0=C y
\end{array} \Longrightarrow\left(\begin{array}{l}
x \\
y
\end{array}\right)=\left(\begin{array}{l}
0 \\
0
\end{array}\right)\right.\right.
$$

where the second and the third implications follow from the facts that $A(X) \cap$ $C(Y)=\{0\}$ and $B^{-1}(0) \cap C^{-1}(0)=\{0\}$, respectively. This completes the proof.

The following is an extension of Du and Pan [1, Theorem 2] to Banach spaces:

Corollary 3. For a given pair $(A, B)$ of operators we have

$$
\bigcap_{C \in \mathcal{L}(Y, X)} \sigma\left(M_{C}\right)=\sigma_{l}(A) \cup \sigma_{r}(B) \cup\left\{\lambda \in \mathbb{C}:(B-\lambda)^{-1}(0) \varsubsetneqq Y /(A-\lambda)(X)\right\} .
$$

Proof. This follows at once from Theorem 2.

The following two corollaries are also immediate results from Theorem 2.

Corollary 4. For a given pair $(A, B)$ of operators we have

$(\sigma(A) \cup \sigma(B)) \backslash(\sigma(A) \cap \sigma(B)) \subseteq \sigma\left(M_{C}\right) \subseteq \sigma(A) \cup \sigma(B) \quad$ for every $C \in \mathcal{L}(Y, X)$.

Proof. The second inclusion comes from Lemma 1. The first inclusion follows from the observation

$M_{C}-\lambda$ is invertible $\Longrightarrow(A-\lambda$ is invertible $\Longleftrightarrow B-\lambda$ is invertible $)$

for each $\lambda \in \mathbb{C}$.

Corollary 5. If $M_{C}$ is Fredholm and if either $A$ or $B$ is Fredholm, then $A$ and $B$ are both Fredholm with

$$
\text { ind } M_{C}=\text { ind } A+\text { ind } B .
$$

Proof. The first assertion follows by applying Theorem 2 with the pair $(\pi(A), \pi(B))$, where $\pi$ is the Calkin homomorphism. The second assertion follows from applying the index product theorem to $(2.1)$.

The equality (5.1) is called the "snake lemma". From this we can also see that if $M_{C}$ is Weyl, in the sense of Fredholm of index zero, and if either $A$ or $B$ is Fredholm, then $A$ is Weyl if and only if $B$ is Weyl. 


$$
\text { 3. The PASsage From } \sigma\left(\begin{array}{ll}
A & 0 \\
0 & B
\end{array}\right) \text { TO } \sigma\left(M_{C}\right)
$$

From Corollary 4 we see that, in perturbing a nilpotent matrix $\left(\begin{array}{ll}0 & C \\ 0 & 0\end{array}\right)$ to $\left(\begin{array}{ll}A & 0 \\ 0 & B\end{array}\right)$, $\sigma\left(M_{C}\right)$ shrinks from $\sigma(A) \cup \sigma(B)$. How much of $\sigma(A) \cup \sigma(B)$ survives? The following theorem provides a clue:

Theorem 6. For a given pair $(A, B)$ of operators we have

$$
\eta\left(\sigma\left(M_{C}\right)\right)=\eta(\sigma(A) \cup \sigma(B)) \quad \text { for every } C \in \mathcal{L}(Y, X),
$$

where $\eta(\cdot)$ denotes the "polynomially convex hull".

Remark. The "polynomially convex hull" (by definition, related to the behaviour of polynomials) is the topological object obtained by "filling in holes".

Proof. By Corollary 4 we have

$$
\sigma\left(M_{C}\right) \subseteq \sigma(A) \cup \sigma(B) \quad \text { for every } C \in \mathcal{L}(Y, X) .
$$

We now claim that

$$
\partial(\sigma(A) \cup \sigma(B)) \subseteq \partial \sigma\left(M_{C}\right),
$$

where $\partial \mathbf{K}$ denotes the topological boundary of $\mathbf{K} \subseteq \mathbb{C}$. Since

$$
\operatorname{int} \sigma\left(M_{C}\right) \subseteq \operatorname{int}(\sigma(A) \cup \sigma(B)),
$$

it suffices to show that $\partial(\sigma(A) \cup \sigma(B)) \subseteq \sigma\left(M_{C}\right)$. Indeed we have

$$
\partial(\sigma(A) \cup \sigma(B)) \subseteq \partial \sigma(A) \cup \partial \sigma(B) \subseteq \sigma_{a p}(A) \cup \sigma_{\delta}(B) \subseteq \sigma_{l}(A) \cup \sigma_{r}(B) \subseteq \sigma\left(M_{C}\right),
$$

where $\sigma_{a p}(\cdot)$ and $\sigma_{\delta}(\cdot)$ denote the approximate point spectrum and the defect spectrum, respectively: the second inclusion follows from the fact that if $T \in \mathcal{L}(Z)$ for a Banach space $Z$, then $\partial \sigma(T) \subseteq \sigma_{a p}(T) \cap \sigma_{\delta}(T)$ and the last inclusion follows from Corollary 3. This proves (6.3). Now the Maximum Modulus Theorem with (6.2) and (6.3) gives (6.1).

The following corollary says that the passage from $\sigma(A) \cup \sigma(B)$ to $\sigma\left(M_{C}\right)$ is the punching of some open sets in $\sigma(A) \cap \sigma(B)$ :

Corollary 7. For a given pair $(A, B)$ of operators we have

$$
\sigma(A) \cup \sigma(B)=\sigma\left(M_{C}\right) \cup W,
$$

where $W$ is the union of certain of the holes in $\sigma\left(M_{C}\right)$ which happen to be subsets of $\sigma(A) \cap \sigma(B)$.

Proof. Theorem 6 says that the passage from $\sigma\left(M_{C}\right)$ to $\sigma(A) \cup \sigma(B)$ is the filling in certain of the holes in $\sigma\left(M_{C}\right)$. But since, by Corollary $4,(\sigma(A) \cup \sigma(B)) \backslash \sigma\left(M_{C}\right)$ is contained in $\sigma(A) \cap \sigma(B)$, the filling some holes in $\sigma\left(M_{C}\right)$ should occur in $\sigma(A) \cap$ $\sigma(B)$. This gives the result.

The following is a generalization of [2, Problem 72].

Corollary 8. If $\sigma(A) \cap \sigma(B)$ has no interior points, then

$$
\sigma\left(M_{C}\right)=\sigma(A) \cup \sigma(B) \quad \text { for every } C \in \mathcal{L}(Y, X) .
$$

In particular if either $A \in \mathcal{L}(X)$ or $B \in \mathcal{L}(Y)$ is a compact operator, then (8.1) holds. 
Proof. The equality (8.1) immediately follows from Corollary 7. The second assertion follows from the fact that the spectrum of a compact operator is at most countable.

One might guess that the closure of each member of $W$ in Corollary 7 is a connected component of $\sigma(A) \cap \sigma(B)$. But this is not the case. See the following:

Example 9. Let $U: \ell_{2} \rightarrow \ell_{2}$ be the unilateral shift and let $K: \ell_{2} \rightarrow \ell_{2}$ be a diagonal operator whose diagonals form a countable dense subset of the annulus $\{z \in \mathbb{C}: 1 \leq|z| \leq 2\}$. Define the operators $A, B$ and $C$ acting on $\ell_{2} \oplus \ell_{2}$ by

$$
A=\left(\begin{array}{cc}
U & 0 \\
0 & K
\end{array}\right), \quad B=\left(\begin{array}{cc}
U^{*} & 0 \\
0 & K
\end{array}\right) \quad \text { and } \quad C=\left(\begin{array}{cc}
1-U U^{*} & 0 \\
0 & 0
\end{array}\right) .
$$

Then we have

$$
\sigma(A)=\sigma(B)=\{z \in \mathbb{C}:|z| \leq 2\} \quad \text { and } \quad \sigma\left(M_{C}\right)=\{z \in \mathbb{C}: 1 \leq|z| \leq 2\},
$$

which shows that the closure of the hole, $\{z \in \mathbb{C}:|z|<1\}$, of $\sigma\left(M_{C}\right)$ is not a component of $\sigma(A) \cap \sigma(B)$.

We now consider another case in which the equality in (8.1) holds. To do this write, for $T \in \mathcal{L}(X)$,

$$
\rho_{\sigma}^{l}(T)=\sigma(T) \backslash \sigma_{l}(T) \quad \text { and } \quad \rho_{\sigma}^{r}(T)=\sigma(T) \backslash \sigma_{r}(T) .
$$

Thus by Corollary 8 and Theorem 2 we can see that the holes in $\sigma\left(M_{C}\right)$ should lie in $\rho_{\sigma}^{l}(A) \cap \rho_{\sigma}^{r}(B)$. Thus we have:

Corollary 10. If $\rho_{\sigma}^{l}(A) \cap \rho_{\sigma}^{r}(B)=\emptyset$ then

$$
\sigma\left(M_{C}\right)=\sigma(A) \cup \sigma(B) \quad \text { for every } C \in \mathcal{L}(Y, X) .
$$

We conclude with an application of Corollary 10.

Corollary 11. Suppose $H$ and $K$ are Hilbert spaces. If either $A \in \mathcal{L}(H)$ is cohyponormal or $B \in \mathcal{L}(K)$ is hyponormal, then

$$
\sigma\left(M_{C}\right)=\sigma(A) \cup \sigma(B) \quad \text { for every } C \in \mathcal{L}(K, H) .
$$

Proof. If $B$ is hyponormal and so is $B-\lambda$ for every $\lambda \in \mathbb{C}$, then $(B-\lambda)^{-1}(0) \subseteq$ $(B-\lambda)^{*-1}(0)$. Thus if $B-\lambda$ is right invertible, then it must be invertible, which implies $\rho_{\sigma}^{r}(B)=\emptyset$. If instead $A$ is cohyponormal, then a similar argument gives $\rho_{\sigma}^{l}(A)=\emptyset$. Thus (11.1) follows from Corollary 10 .

\section{REFERENCES}

1. H.K. Du and J. Pan, Perturbation of spectrums of $2 \times 2$ operator matrices, Proc. Amer. Math. Soc. 121 (1994), 761-776. MR 94i:47004

2. P.R. Halmos, A Hilbert Space Problem Book, Springer, New York, 1973. MR 84e:47001

3. R.E. Harte, Invertibility and Singularity for Bounded Linear Operators, Dekker, New York, 1988. MR 89d:47001

4. R.E. Harte, The ghost of an index theorem, Proc. Amer. Math. Soc. 106 (1989), 1031-1034. MR 92j: 47029

Department of Mathematics Education, Mokwon University, Daejon 301-719, Korea Korea

Department of Mathematics, Woosuk University, Wanju-gun, Cheonbuk 565-800,

Department of Mathematics, Sung Kyun Kwan University, Suwon 440-746, Korea

E-mail address: wylee@yurim.skku.ac.kr 